\title{
Dengue in China: not a passing problem
}

\author{
QIN ChengFeng ${ }^{1,2^{*}} \&$ SHI PeiYong ${ }^{3 *}$ \\ ${ }^{1}$ Department of Virology, Beijing Institute of Microbiology and Epidemiology, Beijing 100071, China; \\ ${ }^{2}$ State Key Laboratory of Pathogen and Biosecurity, Beijing 100071, China; \\ ${ }^{3}$ Wadsworth Center, Albany, New York 12201, USA
}

Received November 11, 2014; accepted November 20, 2014

Citation: Qin CF, Shi PY. Dengue in China: not a passing problem. Sci China Life Sci, 2014, 57: 1230-1231, doi: 10.1007/s11427-014-4783-2

China is experiencing the most severe dengue outbreak since the 1980s. Till 31 October 2014, a total of 44497 laboratory-confirmed dengue cases with six deaths have been reported in mainland China, among which 43031 cases were from Guangdong. Although dengue fever is classified as a notifiable infectious disease according to Chinese Ministry of Health, the public and health care system has not fully appreciated the medical burden of this disease. The unprecedented dengue cases in China have set off the alarm for public health to respond to emerging and re-emerging diseases. A comprehensive strategy is needed to win the battle against dengue and other infectious diseases, including surveillance, diagnostics, vector control, vaccine, and drug development.

Dengue is not a new infectious disease in China. The disease is caused by dengue virus (DENV) infection. It was first described in the medical encyclopaedia "TAI PING SHENG HUI FANG" in Song dynasty. Large outbreaks of dengue fever were well recorded in China during World War II; the disease disappeared for the next 30 years and re-emerged in the 1980s in southern China. For the past few decades, only sporadic outbreaks and imported cases were reported in China. Guangdong province is the major affected areas, and some Guangzhou indigen called it "brokenbone fever" or "mosquito-bite disease".

The current dengue problem in China is a reflection of global situation. Nowadays, dengue is well regarded as the most prevalent mosquito-borne viral disease in humans.

*Corresponding author (email: qincf@bmi.ac.cn; pei_yong.shi@novartis.com)
Half of global population lives at areas at risk of dengue infection. An estimate of 390 million individuals was infected by DENV in more than 100 countries. Dengue activity has increased substantially in many tropical and subtropical countries due to globalization, climate change, environmental deterioration, and social factors. USA, Europe, and Japan have recently suffered their first autochthonous dengue outbreak [1].

Dengue has largely changed its appearance in China, and current situation is more complex than thought. Dengue is a typical mosquito-transmitted viral disease caused by the four serotypes of DENV, which belongs to the genus Flavivirus within the family of Flaviviradae. Aedes aegypti and Aedes albopictus are the major mosquito vectors; the later mosquito is widely distributed in mainland China. Dengue outbreaks in Guangdong are usually seasonal with decreased or no cases in winter. However, people are worrying about the re-emergence of outbreaks in the following years. Aedes albopictus, the primary vector for DENV in Guangdong, can transmit DENV transovarially, thus overwintering mosquitoes may serve as potential source of dengue to initiate a new infection cycle. Importantly, Aedes aegypti, the more effective DENV vector than Aedes albopictus, has been introduced and colonized southern regions of China, including Yunnan and Guangdong provinces [2]. It is likely that DENV will establish its infection in Chinese Aedes aegypti, which can further deteriorate the current situation. Extensive and long-term vector control is needed to mitigate the situation.

After this large outbreak, it is possible that DENV will be 
endemic in southern China, especially in Guangdong. All four serotypes of DENV have been isolated during the past 10 years. The co-circulation of multiple serotypes of DENV increases the risk of severity of disease. Genomic sequence analysis of the newly isolated DENV-2 in Guangzhou during this outbreak demonstrated high homology to the ones isolated in the past decade in Guangdong [3]. Individuals that have been exposed to DENV-2 this year are prone to development of severe disease when infected with a secondary heterotypic DENV infection, due to the phenomena of antibody-dependent enhancement of infection. Severe dengue cases caused by secondary heterotypic DENV have been documented, and the proportion of secondary infection among severe cases is increasing during recent outbreaks in Guangdong (personal communication).

The geographic expansion of dengue disease in China remains to be closely monitored. It is likely that dengue epidemic will continue to spread to many southern provinces, including Guangdong, Yunnan, Guangxi, Hainan, and Fujian. China has to be well prepared. Although the virus has been identified for more than 60 years, no approved vaccine and antiviral is currently available. Promising vaccine and antiviral drug candidates are being developed. The leading CYD dengue vaccine from Sanofi Pasteur is expected to be licensed in 2015 [4]. However, it should be noted that the CYD vaccine has weak protection against DENV-2. It will take some time to introduce such vaccine to China. Although a few compounds have been tested in dengue clinical trials, none of them have shown antiviral activity or clinical benefits in dengue patients [5]. More efforts are needed to develop bona fide antiviral drugs for dengue. The first molecular diagnosis kit targeting DENV genome has just been approved by Chinese Food and Drug
Administration. The point-of-care diagnosis kit targeting the NS1 protein is critically needed in clinics.

Dengue is now turning into a long-term threat in Southern China. There is an urgent need for continued research into the diagnosis, pathogenesis, prevention, and treatment of DENV infections. Like many infectious diseases, pathogens have been evolutionarily evolving with the hosts [6]. To fight them, we humans have to keep sharpening our armory to stay ahead of the war.

1 Guzman MG, Harris E. Dengue. Lancet, 2014, 14: 60572-60579

2 Zhang FC, Zhao H, Li LH, Jiang T, Hong WX, Wang J, Zhao LZ, Yang HQ, Ma DH, Bai CH, Shan XY, Deng YQ, Qin CF. Severe dengue outbreak in Yunnan, China, 2013. Int J Infect Dis, 2014, 27: 4-6

3 Zhao H, Zhao LZ, Jiang T, Li XF, Fan H, Hong WX, Zhang Y, Zhu $\mathrm{Q}$, Ye Q, Tong YG, Cao WC, Zhang FC, Qin CF. Isolation and characterization of dengue virus serotype 2 from the large dengue outbreak in Guangdong, China in 2014. Sci China Life Sci, 2014, 57 : 1149-1155

4 Capeding MR, Tran NH, Hadinegoro SR, Ismail HI, Chotpitayasunondh T, Chua MN, Luong CQ, Rusmil K, Wirawan DN, Nallusamy R, Pitisuttithum P, Thisyakorn U, Yoon IK, van der Vliet D, Langevin E, Laot T, Hutagalung Y, Frago C, Boaz M, Wartel TA, Tornieporth NG, Saville M, Bouckenooghe A. Clinical efficacy and safety of a novel tetravalent dengue vaccine in healthy children in Asia: a phase 3, randomised, observer-masked, placebo-controlled trial. Lancet, 2014, 384: 1358-1365

5 Lim SP, Wang QY, Noble CG, Chen YL, Dong H, Zou B, Yokokawa F, Nilar S, Smith P, Beer D, Lescar J, Shi PY. Ten years of dengue drug discovery: progress and prospects. Antiviral Res, 2013, 100: 500-519

6 Li J, Yu XF, Pu XY, Xie L, Sun YX, Xiao HX, Wang FJ, Din H, Wu Y, Liu D, Zhao GQ, Liu J, Pan JC. Environmental connections of novel avian-origin H7N9 influenza virus infection and virus adaptation to the human. Sci China Life Sci, 2013, 56: 485-492

Open Access This article is distributed under the terms of the Creative Commons Attribution License which permits any use, distribution, and reproduction in any medium, provided the original author(s) and source are credited. 